\title{
Spreading Sequence-Based Non-coherent Sensor Fusion and its Resulting Large Deviation Exponents
}

\author{
Shuangqing Wei \\ Department of Electrical and Computer Engineering \\ Louisiana State University \\ Baton Rouge, LA 70803 \\ Email: swei@ece.lsu.edu
}

\begin{abstract}
To address the coordination issue of sensors communicating with a fusion center, we propose a spreading sequence based non-coherent detection scheme for sensor networks to reduce the coordination between sensors to the largest extent. In this scheme, sensors employ independent spreading sequences to transmit their measurements. Non-coherent detection is conducted at the fusion center where only statistics regarding channel gains and sensor measurement uncertainties are needed. To evaluate the detector's performance, we first derive the large deviation exponents of detection error probabilities and then compare them with the approaches assuming orthogonal channel allocation (e.g.TDMA/FDMA). Numerical and simulation results demonstrate the dependence of large deviation exponent on the asymptotic number of sensors per chip (defined as $c$ ), as well as the better performance of our proposed scheme than the one using non-coherent detection with orthogonal link, for some $c$.
\end{abstract}

Keywords: Spreading Sequence, Large Deviation Exponents and Non-coherent Detection.

\section{INTRODUCTION}

In this paper, we are mainly concerned with how to effectively send data from a set of sensor nodes to a fusion center via a one-hop network. In particular, we are interested in how to reduce the coordination among sensor nodes to the minimum and how fusion performance in terms of binary detection error probability varies with respect to the size of sensornet as a consequence of such reduced coordinations.

In a one-hop sensor network for data fusion, the conventional wisdom was the measured data from each sensor is immediately available at the fusion center. This holds when sensors are traditional radars and a wired link exists between each front end radar and the fusion center. One step further to a more realistic scenario for sensornet with large amount of sensors is to allow orthogonal channel allocation such that signals sent by each sensor go through independent and orthogonal wireless links (e.g. TDMA, FDMA), where distortion and interference are possibly introduced, to the fusion center[1], [2]. However, we can foresee that coordination and resulting overheads are overwhelming in order to achieve exact orthogonalization in large scale sensor networks.

Recently, A. Anandkumar and L. Tong [3] and K. Liu and H. El Gamal and A. Sayeed [4] independently proposed type

\footnotetext{
${ }^{1}$ Shuangqing Wei was supported in part by the Board of Regents of Louisiana under grants LEQSF(2004-07)-RD-A-17 and NSF/LEQSF(2005)PFUND-10.
}

based transmission schemes, where each element of possible quantized outputs is assigned an orthogonal spreading code. At the fusion center, what matters to detector's performance is the superposed signals corresponding to each orthogonal sequence. However, they have shown that when channel experiences zero mean fading, the detection error no longer scales exponentially with respect to the number of sensors.

When we use sensor net to facilitate a binary decision at a fusion center, the ultimate objective is to distinguish between two hypothesis corresponding to the presence or absence of a target. Therefore, there is no necessity to faithfully reconstruct the measurements taken at each sensor node as long as the fusion/detection performance satisfies our need. Inspired by such an observation, we propose a novel spreading sequence based non-coherent data fusion scheme. Sensors multiply independent random spreading sequences with their measurements. There is no need to make these spreading sequences orthogonal with each other. The fusion center does not need to know what sequences sensors have used and what is the channel gain for each sensor-fusion center link. All information required to conduct non-coherent detection/fusion is the statistics of spreading sequences and channel gains, as well as the distributions of measurement error at each sensor. Large system analysis is then invoked to analyze the detector's performance by making the number of users $K$ and the length of spreading sequences $N$ grow to infinity while satisfying $K / N \rightarrow c>0$.

The paper is organized as follows. System model is firstly described in Section II. Then in Section III, a non-coherent data fusion without orthogonal channel allocation is proposed based on a Gaussian approximation. Detection error probabilities of the proposed non-coherent detector are analyzed in terms of large deviation exponents in Section IV. A special case study is conducted in Section V. Finally, conclusions are made in Section VI.

\section{SySTEM MOdEL}

In this section, we describe the system model of a sensor net to assist a binary hypothesis testing at a fusion center. The binary hypothesis testing problem can be formulated as $\mathcal{H}_{0}: \theta=\theta_{0}$ vs. $\mathcal{H}_{1}: \theta=\theta_{1}$, with prior probability $P\left(\mathcal{H}_{0}\right)=\pi_{0}$ and $P\left(\mathcal{H}_{1}\right)=\pi_{1}=1-\pi_{0}$, respectively. We assume $K$ sensors provide measurement to the fusion center. 
Each sensor has a local observation of $Z_{k}, k=1, \cdots, K$ distributed as: $\mathcal{H}_{1}: Z_{k}=m_{S}+W_{k}$ and $\mathcal{H}_{0}: Z_{K}=W_{k}$, where $m_{S}>0$ is a known constant and $\left\{W_{k}, k=1, \cdots, K\right\}$ are independent Gaussian noise distributed as $N\left(0, \sigma_{w, k}^{2}\right)$. Let $X_{k}, k=1, \cdots, K$ denote the processed data output from sensor $k$ to the fusion center, where an aggregated decision is made. The channels between each sensor and the fusion center are assumed to experience independent channel variations $h_{k}$, with mean $\mu_{k}$ and variance $\sigma_{h, k}^{2}$. Due to the large amount of sensors, channel fading variables $h_{k}$ are assumed not available at the receiver end (fusion center), i.e. we are interested in the non-coherent detection.

No orthogonal channel allocation is assumed in the sensor net. Instead, we assume each sensor employs a random spreading code/sequence to carry its transmitted data $X_{k}$ in order to average out the influence of channel uncertainties, as well as local measurement errors. Let $\left\{c_{i, j}, i=1, \cdots, K, j=\right.$ $1, \cdots, N\}$ denote the spreading sequence of length $N$ used by sensor $i$. In this paper, we assume $c_{i, j}$ independently distributed as $\mathcal{N}(0,1)$. Gaussian assumption will simplify our large deviation analysis below. For more general cases of non-Gaussian distributions on $c_{i, j}$, the approaches are more involved and the results will be presented in our future publications [5]. In the sequel, we adopt the matrix $\mathbf{C}$ to represent $\left\{c_{i, j}\right\}$. At the fusion center, the received signals over $N$ slots can be expressed as $Y_{j}=\frac{1}{\sqrt{N}} \sum_{k=1}^{K} h_{k} c_{k, j} X_{k}+n_{j}$ for $j=1, \cdots, N$, where $n_{j} \sim \mathcal{N}\left(0, \sigma_{n}^{2}\right)$ are identically and independently distributed (i.i.d) Gaussian random variables, and $1 / \sqrt{N}$ is a normalization factor such that $\frac{1}{N} \sum_{j=1}^{N} E\left|c_{k, j}\right|^{2}=$ 1 , for $k=1, \cdots, K$. Throughout this paper, we assume $K / N \rightarrow c$ where $0<c<\infty$, as $K \rightarrow \infty$, in order to apply large system analysis.

We are interested in two scenarios regarding the contents associated with $X_{k}$. The first one is an analog transmission scheme in which $X_{k}$ is a scaled version of $Z_{k}$, i.e. $X_{k}=$ $\beta_{A, k} Z_{k}$, where

$$
\text { Case A(nalog): } \beta_{A, k}=\sqrt{P_{s} /\left(\sigma_{w, k}^{2}+\pi_{1} m_{S}^{2}\right)}
$$

is the scaling factor introduced to normalize $E\left|X_{k}\right|^{2}=P_{s}$.

The second approach is to allow each sensor to make local decisions. In this binary detection problem, we have $X_{k}=0$ if the local decision $\hat{\theta}_{k}=\theta_{0}$ is in favor of $\mathcal{H}_{0}$ and $X_{k}=\beta_{B, k}$, otherwise. The scaling factor is

$$
\text { Case B(inary): } \beta_{B, k}=\sqrt{P_{s} /\left(\pi_{0} \hat{P}_{F, k}+\pi_{1} \hat{P}_{D, k}\right)},
$$

where $\hat{P}_{F, k}=\operatorname{Pr}\left[\hat{\theta}_{k}=\theta_{1} \mid \mathcal{H}_{0}\right]$ is the false alarm probability and $1-\hat{P}_{D, k}=\operatorname{Pr}\left[\hat{\theta}_{k}=\theta_{0} \mid \mathcal{H}_{1}\right]$ is the missing error probability.

\section{Non-COHERENT Detection: A GaUssian APPROXIMATION}

In this section, we invoke a Gaussian approximation to propose a non-coherent detector assuming $h_{k}$ and $c_{i, j}$ are not available to the fusion center. Denote $S_{j, K}=$
$\frac{1}{\sqrt{N}} \sum_{k=1}^{K} h_{k} c_{k, j} X_{k}$. It can be shown using Linderberg Theorem [6, pp. 359] that $S_{j, K}$ converges to a Gaussian random variable with zero mean and variance $\sigma_{j, \mathcal{E}}^{2}$ under $\mathcal{H}_{j}$ and case $\mathcal{E} \in\{A, B\}:$

$$
\sigma_{j, \mathcal{E}}^{2}=\lim _{K, N \rightarrow \infty} \frac{1}{N} \sum_{k=1}^{K} \sigma_{h, k}^{2} E\left[X_{k}^{2} \mid \mathcal{H}_{j}, \mathcal{E}\right]
$$

where the limit exits when the distribution of $\sigma_{h, k}^{2}$ and $E\left[X_{k}^{2} \mid \mathcal{H}_{j}\right]$ satisfies certain probability law across space (e.g. sensors are distributed in a given area based on Poisson distribution.) For a special case when $h_{j}, j=1, \cdots, K$ are i.i.d and $W_{j}, j=1, \cdots, K$ are i.i.d, we have

$$
\begin{array}{rr}
\sigma_{1, A}^{2}=c \sigma_{h}^{2} \beta_{A}^{2}\left(\sigma_{w}^{2}+m_{S}^{2}\right) & \sigma_{0, A}^{2}=c \sigma_{h}^{2} \beta_{A}^{2} \sigma_{w}^{2} \\
\sigma_{1, B}^{2}=c \sigma_{h}^{2} \beta_{B}^{2} \hat{P}_{D} & \sigma_{0, B}^{2}=c \sigma_{h}^{2} \beta_{B}^{2} \hat{P}_{F}
\end{array}
$$

where $\sigma_{j, A}^{2}$ and $\sigma_{j, B}^{2}$ are limits of $\sigma_{j}^{2}$ in (1) when sensors employ analog and binary transmissions, respectively, under hypothesis $\mathcal{H}_{j}, j=0,1$. For all other parameters in (2), sensor indexes $k$ are removed to be consistent with the i.i.d assumption on $h_{k}$ and $W_{k}$.

To develop a non-coherent detector, we need to characterize not only the distribution functions of each $Y_{j}$, but also the joint distributions of vector $\left[Y_{1}, \cdots, Y_{N}\right]$. We should be aware that as $N$ and $K$ grow simultaneously to infinity, we cannot apply the Linderberg Central Limit Theorem for fixed dimension random vectors to our problem. However, since our purpose is to come up with a feasible non-coherent detector, we can still argue that when $c_{i, j}$ are i.i.d standard Gaussian random variables, it is a good approximation that $\left\{S_{j}\right\}$ are i.i.d Gaussian random variables with zero mean and variance $\sigma_{i, A}^{2}$ and $\sigma_{i, B}^{2}$ for case $\mathrm{A}$ and $\mathrm{B}$, respectively, under hypothesis $\mathcal{H}_{i}$. As a result, for large $N$ and $K, Y_{j}$ can be approximated as i.i.d Gaussian random variables under hypothesis $\mathcal{H}_{i}, i=0,1$ with distribution $Y_{j} \sim \mathcal{N}\left(0, \sigma_{Y, i, \mathcal{E}}^{2}\right)$, where $\sigma_{Y, i, \mathcal{E}}^{2}=\sigma_{n}^{2}+\sigma_{i, \mathcal{E}}^{2}$ for $\mathcal{E} \in\{A, B\}$. This approximation can therefore lead us to a non-coherent detector for large $N$ and $K$ : when

$$
\frac{\sum_{j=1}^{N}\left|Y_{j}\right|^{2}}{N} \geq \frac{\sigma_{Y, 1, \mathcal{E}}^{2} \sigma_{Y, 0, \mathcal{E}}^{2}}{\sigma_{Y, 1, \mathcal{E}}^{2}-\sigma_{Y, 0, \mathcal{E}}^{2}} \ln \frac{\sigma_{Y, 1, \mathcal{E}}^{2}}{\sigma_{Y, 0, \mathcal{E}}^{2}} \triangleq \eta
$$

the decision at the fusion center is $\tilde{\theta}=\theta_{1}$; and $\tilde{\theta}=\theta_{0}$, otherwise.

\section{ERror EXPONENT OF DETECTION ERror PROBABILITY}

In this section, we study the asymptotic scaling law of detection error probability $P_{F}(N, K)=\operatorname{Pr}\left[\tilde{\theta}=\theta_{1} \mid \mathcal{H}_{0}\right]$ and $P_{M}(N, K)=\operatorname{Pr}\left[\tilde{\theta}=\theta_{0} \mid \mathcal{H}_{1}\right]$ using decision rule in (3). In particular, we study the limit of $-\left(\ln P_{F}(N, K)\right) / K$ and $-\left(\ln P_{M}(N, K)\right) / K$ as $N, K \rightarrow \infty$ while maintaining $\lim (K / N)=c>0$. All results obtained in this Section apply to both Case A and Case B as defined in Section II.

Define moment generating function (MGF) of the proposed non-coherent detection statistic under $\mathcal{H}_{i}, i=0,1$ 
as $\varphi_{N, i}(t)=E_{i}\left[\exp \left(t \sum_{j=1}^{N}\left|Y_{j}\right|^{2}\right)\right]$, where expectation is taken over joint distributions of $X_{k}, h_{k}, n_{j}$ and $c_{k, j}$ for $k=1, \cdots, K$ and $j=1, \cdots, N$. We first investigate the limit of $\lim _{N, K \rightarrow \infty} \frac{1}{N} \ln \varphi_{N, i}(t)$. If the limit exists, which is denoted by $\phi_{Y, i}(t)$, we then compute the Legendre-Fenchel transform of $\phi_{Y, i}(t)$ under $\mathcal{H}_{i}$ [7]:

$$
I_{Y, i}(z)=\sup _{t \in \mathcal{R}}\left\{t z-\phi_{Y, i}(t)\right\},
$$

for $z \in \mathcal{R}$. Gärtner-Ellis Theorem [8] is then employed to derive the large deviation exponent of detection error probability under each hypothesis:

$$
\lim _{N, K \rightarrow \infty} \frac{1}{K} \ln P_{\mathcal{E}}(N, K)=-\frac{I_{Y, i}(\eta)}{c},
$$

where $i=0$ for $\mathcal{E}=F$ and $i=1$ for $\mathcal{E}=M$.

Our approach to calculate $\varphi_{N, i}(t)$ is to first compute the average over the join distributions of independent Gaussian noise $n_{j}$ and spreading sequences while conditioning over given $h_{k}$ and $X_{k}$.

Lemma 1: Given $h_{k}$ and $X_{k}, k=1, \cdots, K$, the conditional MGF of $\varphi_{N, 0}(t)$ is

$$
\begin{aligned}
& \varphi_{N, i}\left(t \mid\left\{h_{k}, X_{k}\right\}\right)=\left(1-2 t \sigma_{n}^{2}\right)^{(-N / 2)} . \\
& \left(1-\frac{2 t}{N\left(1-2 t \sigma_{n}^{2}\right)} \sum_{k=1}^{K}\left|h_{k} X_{k}\right|^{2}\right)^{-N / 2}
\end{aligned}
$$

Proof:

Due to the space limitation, we only provide a brief outline of the proof here. The entire proof of Lemma 1 contains two steps [5]. We first compute the conditional mean of $\varphi_{N, i}(t)$ given $c_{k, j}, h_{k}$ and $X_{k}$, which can be done by exploiting the characteristic function of Chi-square random variables [9]. In the second step, the condition on the random spreading sequences $\left\{c_{k, j}\right\}$ is taken away by utilizing the characteristic function of Wishart distribution [10, pp. 258].

The last step to calculate $\varphi_{N, i}(t)$ is to integrate the RHS term in (6) over the joint distribution of $h_{k}, X_{k}, k=$ $1, \cdots, K$. Since our ultimate objective is to compute the free energy function $\phi_{Y, i}(t)$, i.e. the limit of $\frac{1}{N} \ln \varphi_{N, i}(t)$ as $N, K \rightarrow \infty$, we can use the similar argument in proving Varadhan's Theorem [7] to compute this asymptotic integral. Denote $\tilde{z}=\sum_{k=1}^{K}\left|h_{k} X_{k}\right|^{2} / K$. The expectation of $\left(1-\frac{2 t}{N\left(1-2 t \sigma_{n}^{2}\right)} \sum_{k=1}^{K}\left|h_{k} X_{k}\right|^{2}\right)^{-N / 2}$ over the joint distribution of $\left(h_{k}, X_{k}\right)$ in (6) can be expressed as

$$
\begin{gathered}
E\left[\left(1-\frac{2 t}{N\left(1-2 t \sigma_{n}^{2}\right)} \sum_{k=1}^{K}\left|h_{k} X_{k}\right|^{2}\right)^{-N / 2}\right]= \\
\int_{0}^{1 / a_{t}} \exp \left[-\frac{N}{2} \ln \left(1-a_{t} \tilde{z}\right)\right] Q_{N}(d \tilde{z})= \\
\approx \int_{0}^{1 / a_{t}} \exp \left[N\left(-\frac{1}{2} \ln \left(1-a_{t} \tilde{z}\right)-c I_{\rho, i}(\tilde{z})\right)\right] d \tilde{z}
\end{gathered}
$$

where $a_{t}=2 t c /\left(1-2 t \sigma_{n}^{2}\right), Q_{N}(\tilde{z})$ is the distribution of $\tilde{z}$ and $I_{\rho, i}(\tilde{z})$ is the Legendre-Fenchel transform of the free energy function $\psi_{h, X}^{(i)}(\gamma)$ of $\sum_{k}\left|h_{k} X_{k}\right|^{2}$, i.e. $I_{\rho, i}(\tilde{z})=\sup _{\gamma}\{\tilde{z} \gamma-$ $\left.\psi_{h, X}^{(i)}(\gamma)\right\}$, where

$$
\psi_{h, X}^{(i)}(\gamma)=\lim _{K \rightarrow \infty} \frac{1}{K} \ln E_{\mathcal{H}_{i}}\left[\exp \left(\gamma \sum_{k=1}^{K}\left|h_{k} X_{k}\right|^{2}\right)\right] .
$$

Unfortunately, Varadhan's Theorem cannot be applied here due to the unboundedness of $\ln \left(1-a_{t} \tilde{z}\right)$ for $\tilde{z} \in\left[0,1 / a_{t}\right]$ [7]. Actually, we have to employ the asymptotic expansion of integrals [11] to expand (7) [5] for large $N$ to obtain

$$
\begin{gathered}
\varsigma_{i}(t)= \\
\lim _{N \rightarrow \infty} \frac{1}{N} \ln \int_{0}^{1 / a_{t}} \exp \left[N\left(-\frac{1}{2} \ln \left(1-a_{t} \tilde{z}\right)-c I_{\rho, i}(\tilde{z})\right)\right] d \tilde{z}
\end{gathered}
$$

As a consequence, $\phi_{Y, i}(t)=-\frac{1}{2} \ln \left(1-2 t \sigma_{n}^{2}\right)+\varsigma_{i}(t)$. Having obtained $\phi_{Y, i}(t)$, we can thereafter calculate the conjugate function $I_{Y, i}(z)$ of the free energy function $\phi_{Y, i}(t)$ in (4), which enables us to obtain the scaling law of detection error probabilities as shown in (5).

\section{LARGE DEVIATION EXPONENTS For CASE A With I.I.D $X_{k}$ AND CONSTANT $h_{k}$}

In this section, we solve one special case to obtain $I_{Y, 0}^{(A)}$, the large deviation exponent $I_{Y, 0}$ in (4) for the false alarm error probability when sensors transmit analog data using spreading sequences. In particular, we assume i.i.d sensor measurement uncertainties resulting in i.i.d $X_{k}$, for $k=1, \cdots, K$. For the purpose of simplicity, we further assume $h_{k}=1, k=$ $1, \cdots, K$. When $h_{k}$ are i.i.d, the approach is similar except we need to count the statistics of channel gains in calculating related moment generating functions. Consequently, the free energy function $\psi_{h, X}^{(i)}(\gamma)$ in (8) becomes: $\psi_{h, X}^{(i)}(\gamma)=$ $\ln E_{\mathcal{H}_{i}}\left[\exp \left(\gamma\left|X_{1}\right|^{2}\right)\right]$.

Theorem 1: When sensors employ random spreading sequences to transmit analog data, the false alarm error probability has large deviation exponent

$$
\lim _{K, N \rightarrow \infty} \frac{1}{K} \ln P_{F}(N, K) \approx-\frac{1}{c} I_{Y, 0}^{(A)}(\eta)
$$

where

$$
I_{Y, 0}^{(A)}(\eta)=\frac{1}{2}\left[\frac{\eta}{\sigma_{n}^{2}+c b_{A}}-\ln \frac{\eta}{\sigma_{n}^{2}+c b_{A}}-1\right]
$$

and $b_{A}=\sigma_{w}^{2} \beta_{A}^{2}$.

Proof: The proof of Theorem 1 contains an approximation of $\tilde{z}=\frac{1}{K} \sum_{j=1}^{K}\left|h_{j} X_{j}\right|^{2}$ in (6) by its mean value $b_{A}$, which results in

$$
\lim _{N \rightarrow \infty} \frac{1}{N} \ln \varphi_{N, 0}(t) \approx-\frac{1}{2} \ln \left(1-2 t \sigma_{n}^{2}-2 c t b_{A}\right)
$$

We can then further attain an approximation of

$$
I_{Y, 0}(\eta)=\sup _{t}\left\{t \eta+\frac{1}{2} \ln \left(1-2 t \sigma_{n}^{2}-2 c t b_{A}\right)\right\} .
$$

The supremum is achieved by making $t=\frac{1}{2\left(\sigma_{n}^{2}+c b_{A}\right)}-\frac{1}{2 \eta}$, which then yields (11). 
We should make a remark here that although Theorem 1 is based upon a first order approximation, the error exponent in (10) provide a good indicator as to how the false alarm probability varies with respect to $c$, as shown in numerical results below.

For comparison purpose, we also provide the large deviation exponent of false alarm error probability when orthogonal channel allocations are available (e.g. TDMA, FDMA). Under such an assumption, each sensor sends its measurements through an assigned individual channel to the fusion center where non-coherent detection is conducted. The received signal over the $k$ th channel is thus: $\tilde{Y}_{k}=h_{k} X_{k}+n_{k}$, for $k=1, \cdots, K$, where $h_{k}, X_{k}$ and $n_{k}$ have the same definition as that in Section II. At the fusion center, a similar decision rule as in (3) is employed, i.e. $\sum_{k=1}^{K}\left|\hat{Y}_{k}\right|^{2} / K \geq \hat{\eta}, \theta=\theta_{1}$; otherwise, $\theta=\theta_{0}$, where $\hat{\eta}=\eta(c=1)$ is obtained by letting $c=1$ in (2) and (3).

Theorem 2: For orthogonal transmission, the missing error probability for analog transmission using non-coherent detector has large deviation exponent $\hat{I}_{0}^{(A)}(\hat{\eta})$, i.e.

$$
\lim _{K \rightarrow \infty}-\frac{1}{K} \ln \operatorname{Pr}\left[\sum_{k=1}^{K}\left|\hat{Y}_{k}\right|^{2} / K \geq \hat{\eta} \mid \mathcal{H}_{0}, A\right]=\hat{I}_{0}^{(A)}(\hat{\eta})
$$

where

$$
\hat{I}_{0}^{(A)}(\hat{\eta})=\frac{1}{2}\left[\frac{\hat{\eta}}{\sigma_{n}^{2}+\beta_{A}^{2} \sigma_{w}^{2}}-\ln \frac{\hat{\eta}}{\sigma_{n}^{2}+\beta_{A}^{2} \sigma_{w}^{2}}-1\right]
$$

Proof: The proof is straightforward using Cramer Theorem [5], [7].

In Figure 1(b), we present numerical results for $\hat{I}_{Y, 0}^{(A)}$ in (15) and $I_{Y, 0}^{(A)} / c$ in (11), respectively. In Figure 1(a) are the simulation results on false alarm probability $\left(P_{F}\right)$ versus $K$ for different coefficients $c$. We can see there exists an optimal $c$, the number of users/per degree of freedom, to maximize $I_{Y, 0}^{(A)}$. More importantly, non-coherent detection using spreading sequences to transmit analog data could yield even smaller $P_{F}$ than that for the non-coherent detection with complete orthogonalization for some $c>1$.

\section{CONCLUSION}

We have proposed a novel spreading sequence based noncoherent data fusion scheme for one-hop wireless sensor networks. Large deviation exponents are derived for the detection error probabilities as the number of sensors $K$ and the length of spreading sequences $N$ grow to infinity. Numerical results demonstrate the relationship between error exponent and $c=$ $\lim _{K, N \rightarrow \infty} K / N$.

Due to the space limitation, we restrict ourselves in this paper to the case where Gaussian spreading sequences are used for each sensor's transmission. More general results regarding cases with non-Gaussian random sequences are also available in [5]. We also have investigated the fundamental difference between analog transmission (Case A) and localbinary transmission (Case B) in terms of error exponent, as well as the performance loss as a consequence of non-coherent detection as compared with the coherent case, which will be presented in our future work [5].

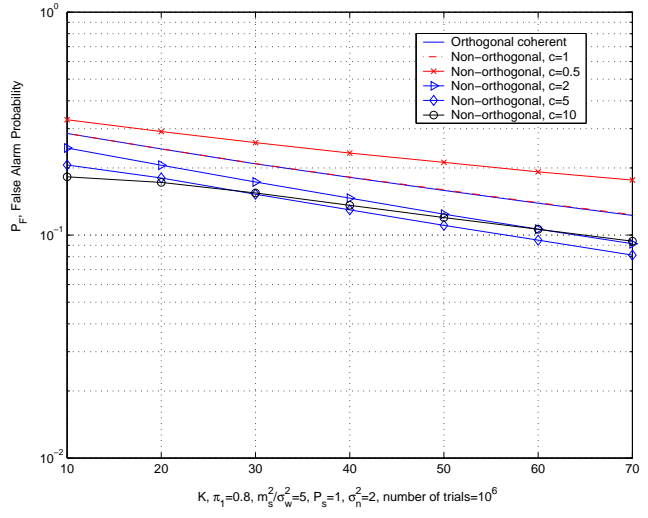

(a) Result 1 on Simulated $P_{F}$.

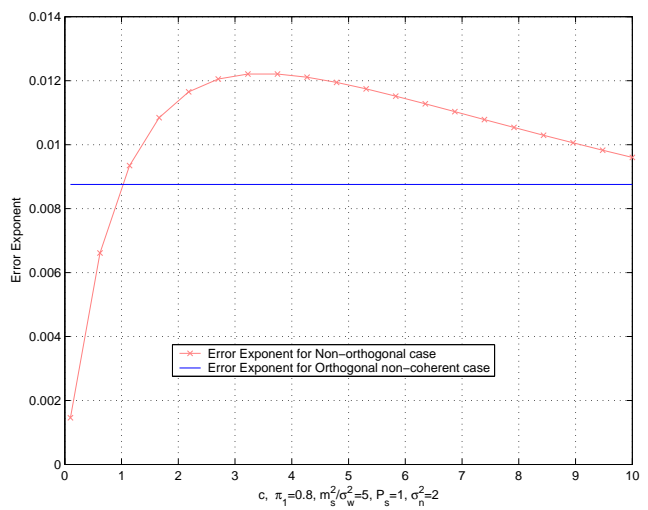

(b) Result 2 on Error Exponent.

Fig. 1. $\pi_{1}=0.8, P_{s}=1, \frac{m_{S}^{2}}{\sigma_{w}^{2}}=5, \sigma_{n}^{2}=2$.

\section{REFERENCES}

[1] J.-F. Chamberland and V.V. Veeravall, "Asymptotic results for decentralized detection in power constrained wireless sensor networks," IEEE JSAC Special Issue on Wireless Sensor Networks, Aug. 2004.

[2] A. Dapos Costa, V. Ramachandran, and A. M. Sayeed, "Distributed classification of gaussian space-time sources in wireless sensor networks," IEEE JSAC Special Issue on Wireless Sensor Networks, Aug. 2004.

[3] A. Anandkumar and L. Tong, "Type-based random access for distributed detection over multiaccess fading channels," IEEE Transactions on Signal Processing, submitted in Dec. 2005 and Revised in June 2006in preparation.

[4] K. Liu, H. El Gamal, and A. Sayeed, "Decentralized inference over multiple access channels," IEEE Transactions on Signal Processing, submitted in Aug. 2005.

[5] S. Wei, "Spreading sequence based data fusion and the resulting large deivation exponents," Tech-Report LSU-ECE-06-20, 2006.

[6] Patrick Billingsley, Probability and Measure, John Wiley \& Sons, New York, 1995, 3rd Edition.

[7] Richard S. Ellis, Entropy, Large Deviations, and Statistical Mechanics, Springer-Verlag, New York, 1985.

[8] James A. Bucklew, Large Deviation Techniques in Decision, Simulation and Estimation, John Wiley \& Sons, New York, 1990.

[9] J. G. Proakis, Digital Communications, McGraw-Hill, Boston, 2001, 4th Edition.

[10] T. W. Anderson, An Introduction to Multivariate Statistical Analysis, John Wiley \& Sons, New York, 2003, 3rd Edition.

[11] R. Wong, Asymptotic Approximations of Integrals, SIAM, Philadelphia, 2001. 